\title{
Potential of Hyper-Kamiokande at some non-Accelerator Physics and Nucleon Decay Searches
}

\author{
Luis Labarga ${ }^{* \dagger}$ \\ Instituto de Física Teórica, UAM/CSIC \\ University Autonoma Madrid \\ E-mail: luis. labarga@uam.es \\ on behalf of The Hyper-Kamiokande proto-Collaboration
}

Hyper-Kamiokande is a next generation water Cherenkov detector consisting of 2 tanks, each with 187 kton fiducial mass, to be built in a staged approach. Hyper-Kamiokande will detect, among others, atmospheric neutrinos as well as neutrinos produced by the upgraded J-PARC accelerator complex, and it will make precision measurements of the neutrino mixing parameters from their combination. Also, Hyper-Kamiokande will enable the search for proton decay and other exotic phenomena with data samples one order of magnitude larger than in current experiments. This talk will describe this physics program.

The European Physical Society Conference on High Energy Physics

5-12 July, 2017

Venice

\footnotetext{
${ }^{*}$ Speaker.

${ }^{\dagger}$ supported by EU H2020-MSCA-RISE-2014-GA641540, SKPLUS.
} 


\section{The Hyper-Kamiokande project}

On the strength of the two Nobel prize winning experiment (Super)Kamiokande and the extremely successful Japanese long baseline neutrino program, a third generation Water Cherenkov experiment based in Japan, Hyper-Kamiokande, is being developed by an international collaboration [1]. It will address the biggest unsolved questions in physics through a multi-decade physics programme that will start in the middle of the next decade.

The Hyper-Kamiokande detector will be hosted in the Tochibora mine (Kamiola, Gifu Prefecture, Japan). The existing accelerator in J-PARC will be steadily upgraded to reach a MW beam by the start of the experiment. Figure 1 shows a global picture, with relevant details, of the experiment as a whole. The experiment will be the largest underground water Cherenkov detector in the world and it will be instrumented with new technology photosensors (PMTs), faster and with higher quantum efficiency than the ones in Super-Kamiokande [2] (see Fig. 2).

Hyper-K is a truly international proto-collaboration with over 60 participating institutions from Brazil, Canada, the United States, France, the United Kingdom, Italy, Korea, Poland, Russia, Spain, and Switzerland, in addition to Japan.

Hyper-K will be a multipurpose neutrino detector with an extremely rich physics program that aims to address some of the most significant questions facing particle physicists today. In this talk we discuss a) oscillation studies from accelerator and atmospheric neutrinos [2] that will refine the neutrino mixing angles and mass squared difference parameters and that will aim to measure a CP-violating phase in the leptonic sector (that will be pivotal in the understanding of the matterantimatter asymmetry in the Universe), and b) the search for nucleon decays thus probing one of the key tenets of Grand Unified Theories.

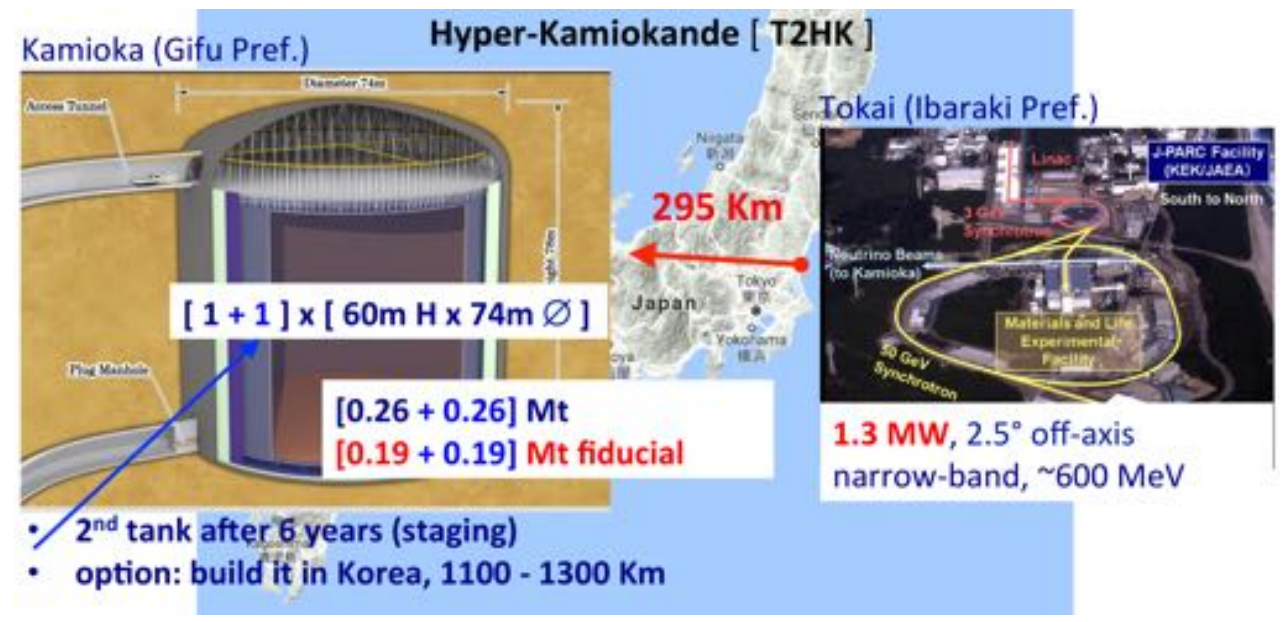

Figure 1: The Hyper-Kamiokande project and T2HK long base line experiment.

\section{Photosensors}

The Hyper-K's inner detector (ID) photosensor, Hamamatsu R12860-HQE, was newly developed based on the well established and reliable design of Super-K's 50cm R3600 PMT by Hamamatsu Photonics K.K. and KamLAND's Hamamatsu R7250. It features a significantly faster time 
response, better charge resolution and a higher detection efficiency with a stable mechanical structure, compared to the existing large aperture PMTs. These properties have a particularly relevant impact on the achievable solar- $v$ physics, neutron tagging by proton capture and the reconstruction of the proton-decay mode $p \rightarrow \bar{v} K^{+}$.

The Quantum Efficiency $(\mathrm{QE})$ reaches $\sim 31 \%$ at $\approx 400 \mathrm{~nm} ; 41 \%$ larger than the conventional R3600 (see Fig. 2-left). Figure 2-middle-left shows the detection efficiency, twice as high as R3600's, and its good uniformity over the whole PMT surface in spite of the asymmetric dynode structure. The single photoelectron pulse in a HQE B\&L PMT shows a 35\% resolution in $\sigma$ that is to be compared to the $50 \%$ of the Super-K PMT (Fig. 2-middle-right). The peak-to-valley ratio is about 4 . The time resolution for single photo-electrons is $1.1 \mathrm{~ns}$ in $\sigma$ for the fast left side of the transit time peak and $4.1 \mathrm{~ns}$ at FWHM (Fig. 2-right). The nominal gain is 107 and it can be adjusted for several factors in a range between $1500 \mathrm{~V}$ to $2200 \mathrm{~V}$. All these features impact very positively the reconstruction performance of events in Hyper-K.

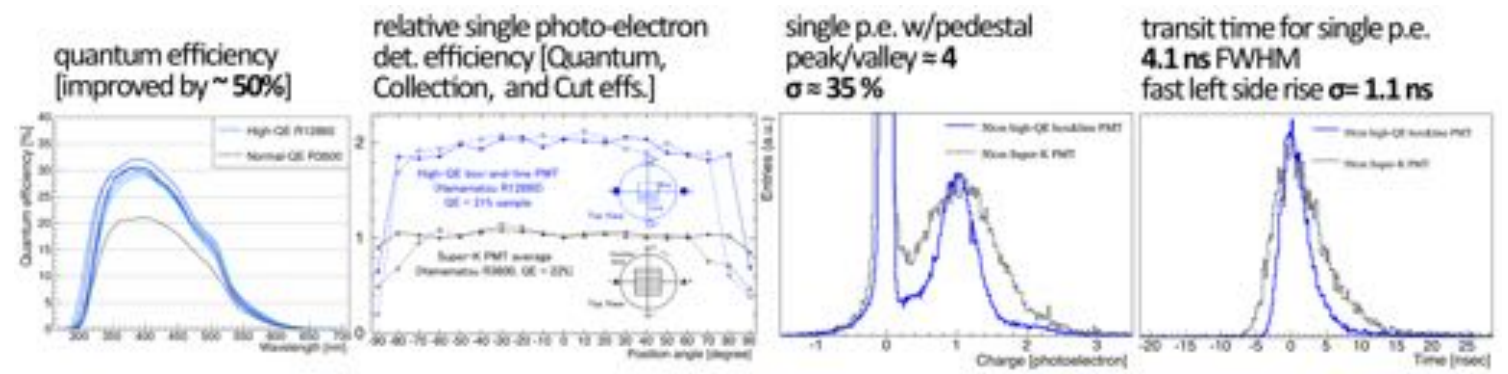

Figure 2: Comparison of performance between the HK's high-QE R12860 (blue lines) and SK's normal R3600 (black lines). See text for details.

\section{Oscillation Analyses}

As atmospheric neutrinos span both low and high energies as well as long and short path lengths, they are sensitive to all parameters in the PMNS mixing paradigm. That being said, the most apparent oscillation features are driven by the so-called atmospheric mixing parameters, $\theta_{23}$ and $\Delta m_{32}^{2}$, and they induce a deficit of observed upward-going $v_{\mu}$ interactions at predominantly multi-GeV energies as these neutrinos oscillate into primarily unobserved $v_{\tau}$. However, now that the value of $\theta_{13}$ is known to be non-zero the presence of matter effects on atmospheric neutrinos that traverse the earth makes important contributions to this picture. Matter-induced parametric oscillations in the energy range 2-10 GeV lead to significant enhancement of either the $v_{\mu} \rightarrow v_{e}$ or the $\bar{v}_{\mu} \rightarrow \bar{v}_{e}$ appearance probability for upward-going neutrinos depending upon the mass hierarchy. For the normal (inverted) hierarchy neutrino (anti-nuetrino) oscillations are enhanced (see Fig. 3-left). This enhancement leads to appearance probabilities around 50\% for both hierarchies. The separation of atmospheric neutrino data into neutrino-like and antineutrino-like subsets can therefore be used to extract the hierarchy signal. Importantly, the features of these oscillations are also a strong function of $\theta_{23}$ and, to a lesser extent, the value of $\delta_{C P}$. It should be noted that these matter effects affect both the appearance and disappearance, $v_{\mu} \rightarrow v_{\mu}$, channels, enabling mass hierarchy sensitivity in both the $v_{e}$-like and $v_{\mu}$-like data at Hyper-K.

Figure 3-middle shows the expected sensitivity to the neutrino mass hierarchy sensitivity and Figure 4-left shows that to the octant as a function of the true value of $\sin ^{2} \theta_{23}$ for a single detec- 
tor after 10 years (a 1.9 Mton-year exposure). The blue (red) band denotes the normal (inverted) hierarchy and the uncertainty from $\delta_{C P}$ is shown by the width of the band. For comparison, Figure 4-middle shows the latter from a beam neutrino measurement with reactor constraint.

Figure 3-left shows the expected sensitivity to the mass hierarchy as a function of time assuming $\sin ^{2} \theta_{23}=0.4$ (triangle), 0.5 (circle), and 0.6 (square) from a combined analysis of atmospheric and accelerator neutrinos data at Hyper-K. Similarly Fig. 4-right shows the expected octant resolution sensitivity for $\sin ^{2} \theta_{23}=0.45$ (solid) and 0.55 (dashed) as a function of time. Error bars indicate the uncertainty from $\delta_{C P}$. Blue (red) colors denote the normal (inverted) hierarchy.
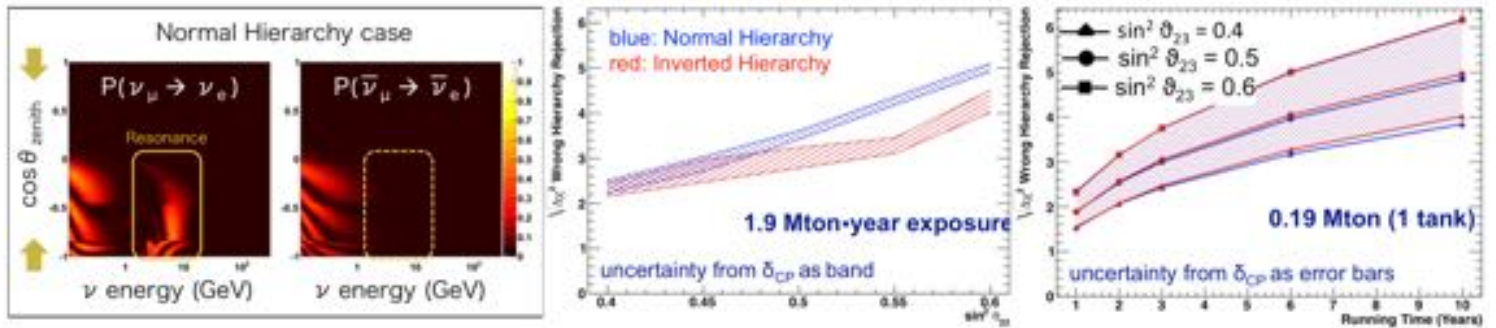

Figure 3: Sensitivity to Mass Hierarchy. See text for details.
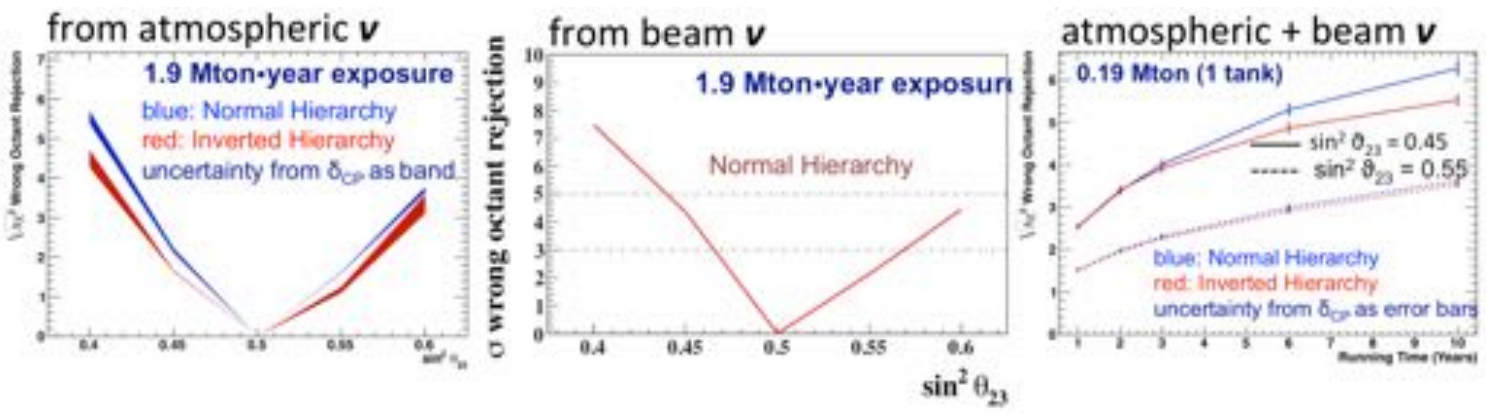

Figure 4: Sensitivity to $\theta_{23}$. See text for details.

\section{Grand Unification}

A primary goal of Hyper-Kamiokande is the thorough experimental study of Grand Unification by the search and maybe observation and discovery of a nucleon decay signal. One of the strengths of the water Cherenkov technology is its sensitivity to a wide variety of decay modes. We show here Hyper-K's expected sensitivity to the two flagship proton decay modes, $p \rightarrow \bar{v} K^{+}$that is feature of supersymmetric grand unified theories, and $p \rightarrow e^{+} \pi^{0}$, favored mode of many GUT models.

Searching for the $K^{+}$in a water Cherenkov detector is complicated by the fact that it emerges from the decay with a momentum of only $340 \mathrm{MeV} / \mathrm{c}$, well below the threshold for light production of $749 \mathrm{MeV} / \mathrm{c}$. As a result the $K^{+}$must be identified by its decay products: $K^{+} \rightarrow \mu^{+} v(64 \%$ branching fraction) and $K^{+} \rightarrow \pi^{+} \pi^{0}$ (21\% branching fraction). Since both of these modes are two body decays the outgoing particles have monochromatic momenta. Fig. 5-middle-left shows the expectations, along the estimated atmospheric background for the first case, and Fig. 5-middle for the second. On the other hand, the 12 ns lifetime of the kaon makes it possible to observe prompt $\gamma$-ray emission produced when the proton hole leftover from a bound proton decay is filled by the de-excitation of another proton. For ${ }^{16} \mathrm{O}$ nuclei the probability of producing a $6 \mathrm{MeV} \gamma$ from such 
a hole is roughly $40 \%$, making this a powerful tool for identifying the $K^{+}$decay products and rejecting atmospheric neutrino backgrounds (see Fig. 5-left). Three methods, each targeting these different aspects of the $K^{+}$decay, are used to search for $p \rightarrow \bar{v} K^{+}$events (see [3] for the details of the analyses). Figure 5-middle-right shows the $3 \sigma p \rightarrow \bar{v} K^{+}$discovery potential and the sensitivity to this mode mode at $90 \%$ C.L. in case of no signal (Fig. 5-middle-right), as a function of run time for HK as well as for other planned experiments.
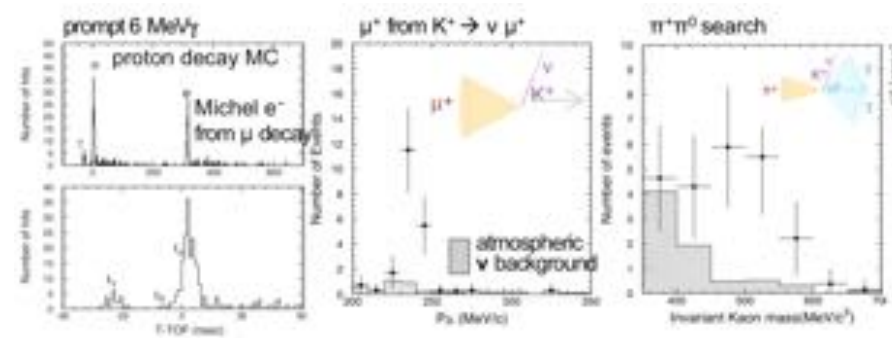

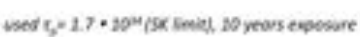
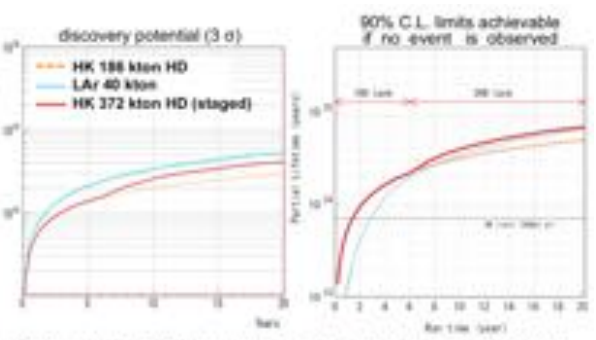

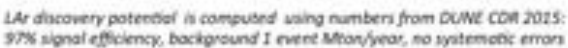

Figure 5: Around the $p \rightarrow \bar{v} K^{+}$mode of proton decay. See text for details.

Experimentally, the $p \rightarrow e^{+} \pi^{0}$ decay mode has a very clean event topology, with no invisible particles in the final state, being possible to fully reconstruct the proton's mass from its decay products. As it is a two body process the total momentum of the recoiling system should be small. Figure 6-left shows the expected distribution of that variable for both signal events and atmospheric $v$ backgrounds. Figure 6-middle shows the $3 \sigma p \rightarrow e^{+} \pi^{0}$ discovery potential and, (right) the sensitivity to this mode at $90 \%$ C.L. in case of no signal, as a function of run time, for HK as well as for other planned experiments.
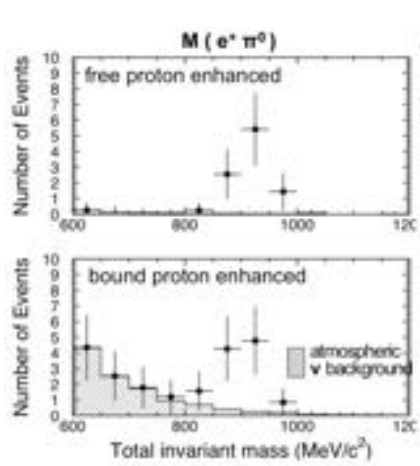

used $\tau_{p}=1.7 \cdot 10^{34}$ (SK limit) 10 yeors exposure
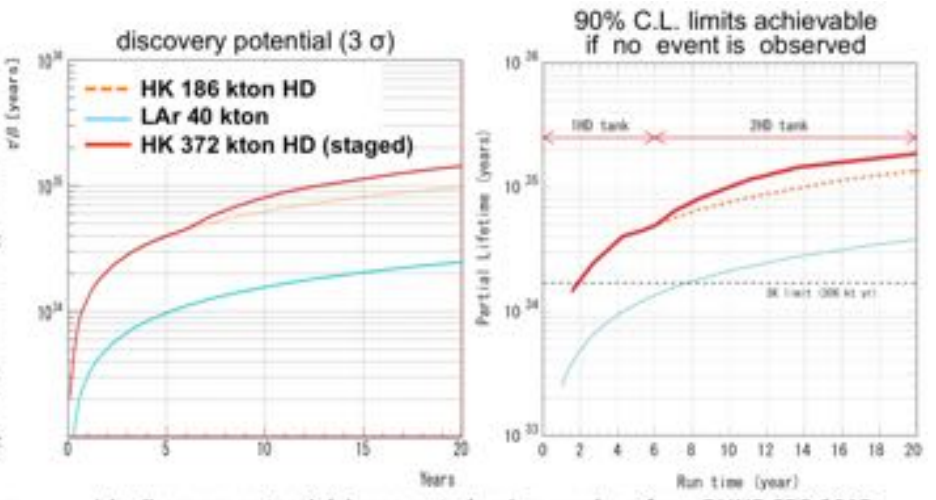

LAr discovery potential is computed using numbers from DUNE CDR 2015: 97\% signal efficiency, background 1 event Mton/year, no systematic errors

Figure 6: Around the $p \rightarrow e^{+} \pi^{0}$ mode of proton decay. See text for details.

5. Conclusions. Hyper-Kamiokande is the next generation Megaton-like, neutrino and nucleon decay experiment. We have presented some of its superb physics expectations. Remarkably, HyperKamiokande will provide by far the most complete experimental exploration of Grand Unification within the next decades.

\section{References}

[1] Hyper-Kamiokande proto-Collab.; K. Abe et al., KEK Preprint 2016-21, ICRR-Report 701-2016-1 
[2] B. Richards, The Hyper-Kamiokande Experiment; talk at this Conference; contribution \#798 J. Migenda, Astroparticle Physics in HK; talk at this Conference; contribution \#518;

[3] Super-Kamiokande Collab.; K. Abe et al., Phys. Rev. D90, 072005 (2014) 